\title{
CO-029 is overexpressed in gastric cancer and mediates the effects of EGF on gastric cancer cell proliferation and invasion
}

\author{
HONGYU ZHU ${ }^{1}$, YULIAN WU ${ }^{2}$, WEN ZHENG ${ }^{1}$ and SHILIU LU ${ }^{1}$ \\ ${ }^{1}$ Zhejiang University School of Medicine; ${ }^{2}$ The Second Affiliated Hospital of Zhejiang University \\ School of Medicine, Hangzhou, Zhejiang 310000, P.R. China
}

Received August 20, 2014; Accepted December 22, 2014

DOI: 10.3892/ijmm.2015.2069

\begin{abstract}
Tetraspanins are cell-surface glycoproteins and have received attention recently as both suppressors and promoters of metastasis. CO-029 is a member of the tetraspanin family and is implicated to be a metastasis-promoting tetraspanin in some cancers. However, the role of CO-029 in gastric cancer remains unexplored. The present study aimed to investigate the expression of CO-029 in gastric cancer tissues and to determine whether CO-029 is involved in the effects of epidermal growth factor (EGF) on gastric cancer cell proliferation and invasion. We collected clinical samples and found that the expression of CO-029 was increased both at the mRNA level and protein level in gastric cancer tissues in comparison to normal and tumor-adjacent tissues, as demonstrated by RT-qPCR and western blot analysis, respectively. Furthermore, we performed an in vitro experiment using AGS cells and observed that EGF promoted AGS cell proliferation and enhanced the invasion ability of the AGS cells, as shown by MTT assay and cell invasion assay, respectively. To the best of our knowledge, our results reveal for the first time, that $\mathrm{CO}-029$ expression was affected by EGF in a concentration- time-dependent manner. The knockdown of CO-029 attenuated the effects of EGF on gastric cancer cell proliferation and invasion. These findings suggest that $\mathrm{CO}-029$ is an oncogene in human gastric cancer and that CO-029 at least partially mediates the effects of EGF on gastric cancer cell proliferation and invasion. Our data may provide a novel target for therapeutic intervention in human gastric cancer.
\end{abstract}

\section{Introduction}

Gastric cancer is one of the leading causes of cancer-related mortality worldwide (1). Progress in gastric cancer has been slow, but steady (2). The majority of gastric cancer patients

Correspondence to: Professor Yulian Wu, The Second Affiliated Hospital of Zhejiang University School of Medicine, 88 Jiefang Road, Hangzhou, Zhejiang 310000, P.R. China

E-mail:wuyulian62@163.com

Key words: CO-029, gastric cancer, proliferation, invasion have massive metastatic spread at the time of initial diagnosis (3). Patients with advanced gastric cancer have a poor prognosis (4), and this results in approximately 800,000 deaths worldwide annually (5). Therefore, it is necessary to investigate the molecular mechanisms underlying the invasive capacity of gastric cancer so as to enhance the curative effects against gastric cancer.

Tetraspanins are cell-surface glycoproteins that are characterized by the presence of 4 hydrophobic domains (6-8). They form protein complexes with integrins and mediate signal transduction events that play key roles in the regulation of cell growth, activation, development and motility (9-13). Tetraspanins have received attention as both suppressors and promoters of metastasis (14-16).

CO-029 is a member of the tetraspanin family. CO-029 has been implicated to be a metastasis-promoting tetraspanin in certain types of cancer, including colon carcinoma (17), esophageal carcinoma (18), pancreatic cancer (19) and hepatocellular carcinoma (20). However, to the best of our knowledge, studies on the functions of $\mathrm{CO}-029$ in gastric cancer cell proliferation and invasion are limited.

Epidermal growth factor (EGF) is a peptide of 53 amino acid residues, it binds to the epidermal growth factor receptor (EGFR) and initiates the cascade of intracellular signaling pathways. Therefore, EGF is involved in a variety of physiological and pathologic processes, including cell proliferation, apoptosis, migration, survival and angiogenesis (21-23). However, the potential molecular mechanisms underlying the effect of EGF on gastric cancer cell growth have not yet been fully elucidated.

Hence, in the present study, we investigated the expression of CO-029 in gastric cancer tissues in order to determine whether CO-029 is involved in the effects of EGF on gastric cancer cell proliferation and invasion.

\section{Materials and methods}

Tissues. Approval for the present study was obtained from the Ethics Committee of The Second Affiliated Hospital of Zhejiang University School of Medicine, Hangzhou, China and informed consent was obtained from all patients prior to enrollment in this study. Gastric cancer tissues, tumor-adjacent tissues and normal gastric tissues were surgically resected from 32 patients with primary gastric cancer. None of the 32 patients had received 
chemotherapy or radiotherapy prior to surgery. The specimens were immediately used for mRNA and protein extraction.

Cell culture. AGS cells purchased from the American Type Culture Collection (ATCC, Manassas, VA, USA) were cultured in Dulbecco's modified Eagle's medium with $10 \%$ fetal bovine serum (FBS) (both from Invitrogen, Carlsbad, CA, USA) in the presence of $5 \% \mathrm{CO}_{2}$ at $37^{\circ} \mathrm{C}$. The culture medium was replaced every 2-3 days. EGF was purchased from Sigma (St. Louis, MO, USA) and dissolved in PBS at different concentrations $(5,10,25,50$ and $100 \mathrm{ng} / \mathrm{ml})$ for cell stimulation.

Reverse transcription-quantitative (real-time) PCR (RT-qPCR). Total RNA was extracted using TRIzol reagent (Invitrogen). Total RNA $(2 \mu \mathrm{g})$ was used to perform reverse transcription using the RevertAid First Strand cDNA Synthesis kit (Fermentas, Vilnius, Lithuania). Real-time PCR was performed on a 7900 real-time PCR system using the SYBR-Green PCR kit (both from Applied Biosystems, Foster City, CA, USA). All procedures were carried out according to the manufacturer's instructions. The $\mathrm{Ct}$ value was calculated using the $\Delta \Delta \mathrm{Ct}$ method.

Western blot analysis. The protein samples from the tissues and cultured cells were isolated using a total protein extraction kit (Sangon Biotech, Shanghai, China) following the manufacturer's instructions. Protein concentrations were examined using the BCA method with reagents from Pierce (Rockford, IL, USA). Protein samples were separated by $10 \%$ sodium dodecyl sulphatepolyacrylamide gel electrophoresis (SDS-PAGE) and transferred onto polyvinylidene difluoride membranes. Subsequently, the membranes were incubated in blocking solution (Tris-buffered saline containing $5 \%$ non-fat milk and $0.05 \%$ Tween-20) at $4{ }^{\circ} \mathrm{C}$ overnight. The blots were probed with the primary antibodies [rabbit polyclonal to CO-029 (sc-292058), 1:400 dilution; rabbit polyclonal to $\beta$-actin (sc-130656), 1:400 dilution] followed by IgG-horse radish peroxidase (HRP)-conjugated secondary antibody [goat anti-rabbit IgG-HRP (sc-2004), 1:2,000 dilution] (all from Santa Cruz Biotechnology, Inc., Santa Cruz, CA, USA). The signal was detected using an ECL western blotting kit (Pierce) and the LabWork 4.0 software (UVP Inc., Upland, CA, USA) was used for semi-quantitative analysis. $\beta$-actin was used as a loading control.

Transfection. For siRNA transfection, the cells were seeded into 6 -well plates and allowed to grow to approximately $70 \%$ confluence. The cells were then transfected with $2 \mu \mathrm{M} \mathrm{CO}-029$ siRNA or scramble control siRNA using Lipofectamine 2000 (Invitrogen) following the manufacturer's instructions. Following incubation with the transfection mixtures for $4 \mathrm{~h}$, the medium was removed and the cells were incubated with fresh Dulbecco's modified Eagle's medium containing 10\% FBS for $24 \mathrm{~h}$. Scramble control siRNA (Scr)-transfected cells were incubated with PBS or EGF; CO-029 siRNA (Si)-transfected cells were incubated with EGF.

MTT assay. Cell proliferation was determined by MTT assay. The cells were seeded into 96-well plates and EGF was added to the medium. Following treatment for $12,24,48$ and $72 \mathrm{~h}$, the cells were incubated with $10 \mu \mathrm{l}$ MTT (Sigma) at $37^{\circ} \mathrm{C}$ for $4 \mathrm{~h}$. The medium was then removed and the cells were incubated

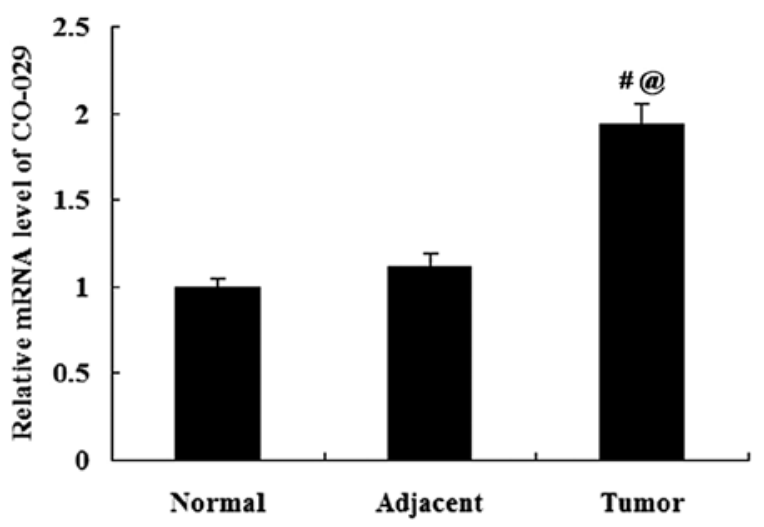

Figure 1. Relative mRNA level of CO-029 in human normal gastric tissues, gastric cancer tissues and tumor-adjacent tissues. ${ }^{\text {} P}<0.01$ vs. normal group; ${ }^{\circledR}$ $\mathrm{P}<0.01$ vs. adjacent group.

with $200 \mu$ l dimethyl sulfoxide to solubilize the formazan crystals. Untreated cells were used as the contrls. The absorbance at $570 \mathrm{~nm}$ was measured using a microplate reader (Molecular Devices, Sunnyvale, CA, USA).

Cell invasion assay. Cells in the blank group were incubated with Dulbecco's modified Eagle's medium containing 10\% FBS. PBS was added to the control group as the vehicle control. Cell invasion ability was evaluated using Transwell inserts coated with Matrigel. Transwell inserts (Corning, Inc., Corning, NY, USA) were coated with Matrigel matrix (BD Biosciences, Franklin Lakes, NJ, USA) at a final concentration of $200 \mu \mathrm{g} / \mathrm{ml}$. The cell plates with the coated inserts were incubated at $37^{\circ} \mathrm{C}$ for $2 \mathrm{~h}$. Cell suspension was prepared in serum-free medium containing $5 \times 10^{4}$ cells $/ \mathrm{ml}$ and added to the upper chambers. A total of $1 \mathrm{ml}$ of cell medium containing $10 \%$ FBS was added to the lower chambers. The cell invasion chambers were incubated overnight in a $37^{\circ} \mathrm{C}, 5 \% \mathrm{CO}_{2}$ atmosphere. A cotton swab was used to gently remove the non-invaded cells. The cells on the lower surface of the membrane were fixed in $95 \%$ ethanol and then stained with hematoxylin for $10 \mathrm{~min}$. The number of invaded cells was evaluated by counting the cells under an inverted microscope (Nikon, Tokyo, Japan).

Statistical analysis. Descriptive data are presented as the means \pm standard deviation (SD). Differences between 2 groups were evaluated using the Student's t-test. A P-value $\leq 0.05$ was considered to indicate a statistically significant difference.

\section{Results}

Expression of $\mathrm{CO}-029$ in human gastric cancer tissues. The expression of CO-029 in human normal gastric tissues, gastric cancer tissues and tumor-adjacent tissues was analyzed by RT-qPCR and western blot analysis. The mRNA expression of CO-029 did not differ significantly between the normal gastric tissues and the tumor-adjacent tissues $(\mathrm{P}>0.05)$; however, the CO-029 mRNA expression was significantly increased in the gastric cancer tissues compared with both the normal gastric tissues and the tumor-adjacent tissues $(\mathrm{P}<0.01$; Fig. 1$)$. The results from western blot analysis revealed that $\mathrm{CO}-029$ protein expression was detectable in the human normal gastric 

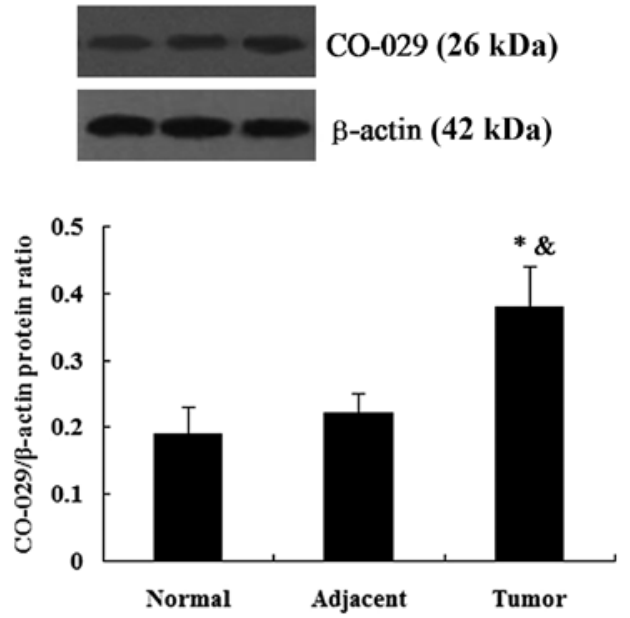

Figure 2. Relative protein level of CO-029 in human normal gastric tissues, gastric cancer tissues and tumor-adjacent tissues. " $\mathrm{P}<0.05$ vs. normal group; ${ }^{\&} \mathrm{P}<0.05$ vs. adjacent group.

tissues, gastric cancer tissues and tumor-adjacent tissues. Similar to the results obtained for the mRNA expression, the gastric cancer tissues showed an upregulated protein expression of CO-029 in comparison to the normal gastric tissues and tumor-adjacent tissues $(\mathrm{P}<0.051$; Fig. 2$)$.

Effect of EGF on CO-029 protein expression in AGS cells. The cells were treated with various concentrations of EGF for $24 \mathrm{~h}$, and western blot analysis was then performed to examine the effect of EGF on CO-029 protein expression. We observed that treatment with EGF increased the expression level of CO-029 in a dose-dependent manner. EGF at the concentration of 5 and $10 \mathrm{ng} / \mathrm{ml}$ did not seem to affect $\mathrm{CO}-029$ protein expression. However, EGF at the concentrations of 25 to $100 \mathrm{ng} / \mathrm{ml}$ increased the expression level of $\mathrm{CO}-029$ and its expression level reach a peak value at $100 \mathrm{ng} / \mathrm{ml}(\mathrm{P}<0.01)$ (Fig. 3A).

The cells were also treated with $100 \mathrm{ng} / \mathrm{ml}$ EGF for 6 to $48 \mathrm{~h}$ (Fig. 3B) and the results demonstrated that EGF affected CO-029 protein expression in a time-dependent manner. EGF at a concentration of $100 \mathrm{ng} / \mathrm{ml}$ increased the expression level of CO-029 following 12 to $48 \mathrm{~h}$ of treatment $(\mathrm{P}<0.01)$. However, there was no significant difference observed between the 6-h treatment group and the control group $(\mathrm{P}>0.05)$.

Effect of EGF on cell proliferation. The cells were treated with $100 \mathrm{ng} / \mathrm{ml} \mathrm{EGF}$ for 12 to $72 \mathrm{~h}$, and MTT assay was then performed to determine the effect of EGF on cell proliferation. We found that EGF promoted cell growth, and the cells treated with EGF proliferated at a higher rate compared with the untreated controls (Fig. 4).

Effect of EGF on cell invasion. To determine the effect of EGF on the invasion ability of the cells, the cells were treated with $100 \mathrm{ng} / \mathrm{ml}$ EGF for $24 \mathrm{~h}$ and Transwell-Matrigel invasion assay was then performed. Cell invasion assay revealed that the cells treated with EGF showed an enhanced invasion ability in comparison to the untreated controls $(\mathrm{P}<0.01)$ (Fig. 5).

Suppression of CO-029 expression attenuates the effects of EGF on cell proliferation and invasion. The cells transfected
$\mathbf{A}$
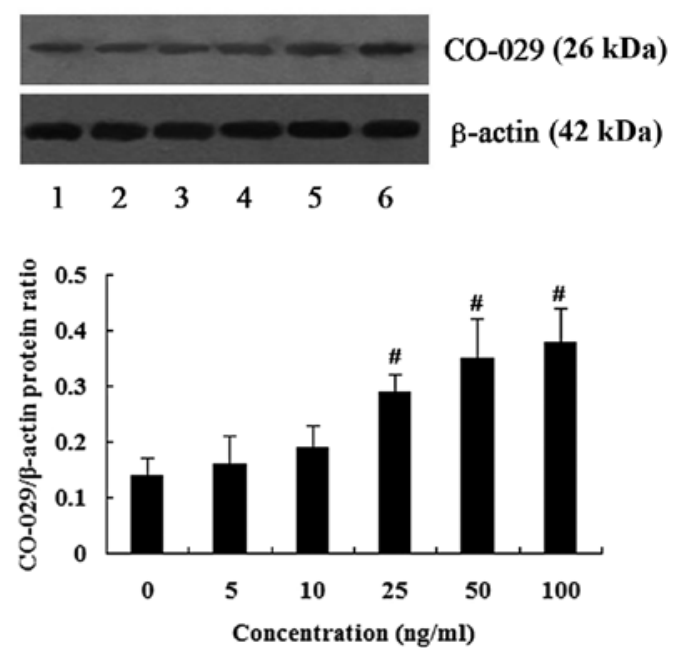

$\mathbf{B}$
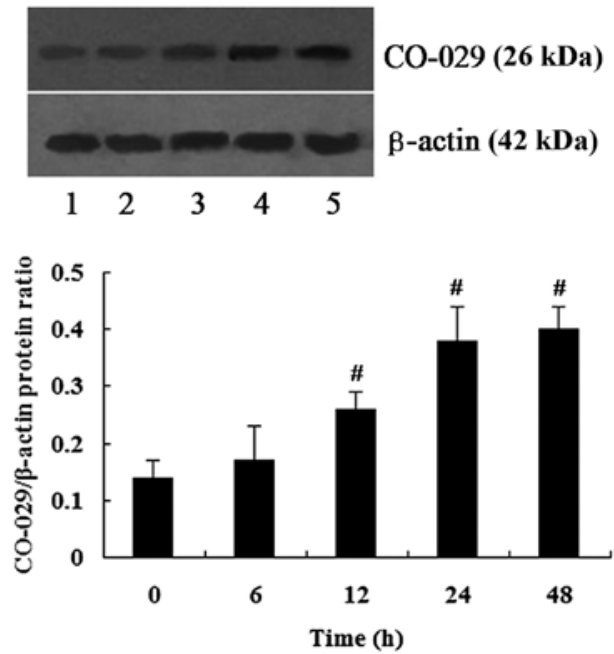

Figure 3. Effect of epidermal growth factor (EGF) on CO-029 protein expression in AGS cells. (A) The concentration-dependent effect of EGF on CO-029 protein expression. Lanes 1-6 represent cells that were treated with $0,5,10$, 25, 50 and $100 \mathrm{ngl} / \mathrm{ml} \mathrm{EGF}$, respectively. ${ }^{~} \mathrm{P}<0.01$ vs. treatment with $0 \mathrm{ng} /$ ml. (B) The time-dependent effect of EGF on CO-029 protein expression. Lanes 1-5 represent cells that were treated with EGF for 0,6, 12, 24 and $48 \mathrm{~h}$, respectively. ${ }^{*} \mathrm{P}<0.01$ vs. treatment for $0 \mathrm{~h}$.

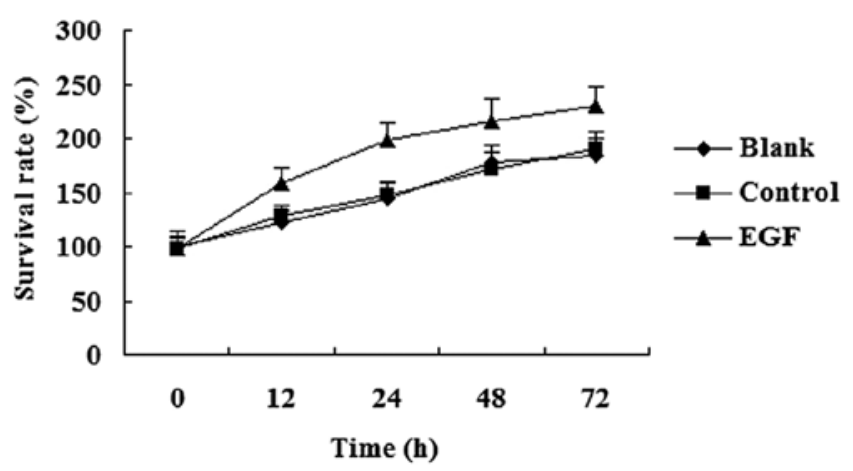

Figure 4. Effect of epidermal growth factor (EGF) on cell proliferation determined by MTT assay.

with CO-029 siRNA (Si) or scramble control siRNA (Scr) were treated with $100 \mathrm{ng} / \mathrm{ml}$ EGF for $24 \mathrm{~h}$ followed by the 


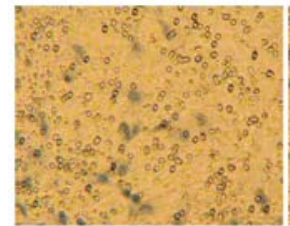

Blank

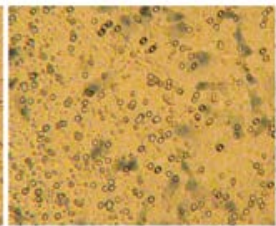

Control

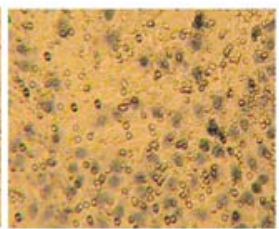

EGF

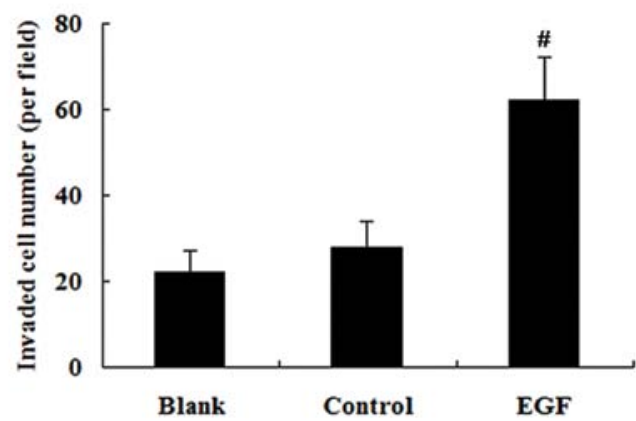

Figure 5. Effect of epidermal growth factor (EGF) on cell invasion ability determined by Transwell-Matrigel invasion assay. Magnification, $\mathrm{x} 400 .{ }^{\#} \mathrm{P}<0.01$ vs. control group. Cells in the blank group were incubated with Dulbecco's modified Eagle's medium containing 10\% FBS. PBS was added to the control group as the vehicle control.
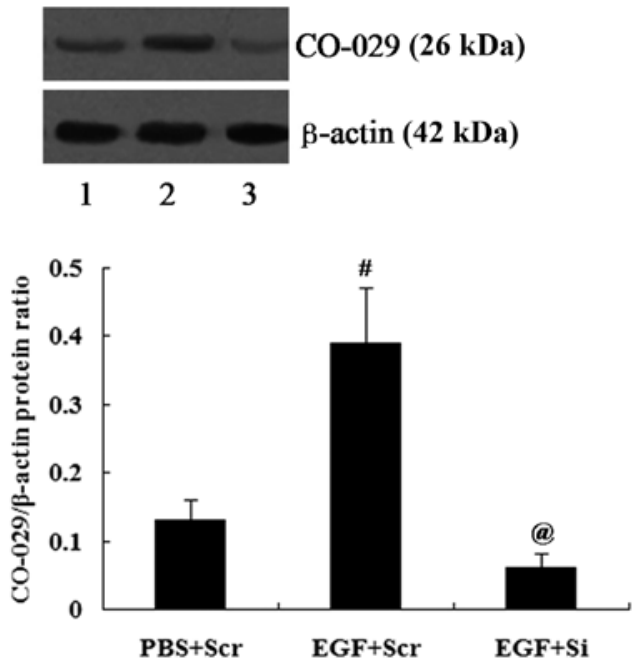

Figure 6. Protein expression of CO-029 in CO-029 siRNA (Si)- or scramble control siRNA (Scr)-transfected cells with or without epidermal growth factor (EGF) stimulation. Lane 1, PBS + Scr group; lane 2, EGF + Scr group; lane 3 , $\mathrm{EGF}+\mathrm{Si}$ group. ${ }^{\#} \mathrm{P}<0.01$ vs. $\mathrm{PBS}+\mathrm{Scr}$ group; ${ }^{\circledR} \mathrm{P}<0.01$ vs. $\mathrm{EGF}+\mathrm{Scr}$ group.

determination of CO-029 protein expression levels by western blot analysis. CO-029 expression was successfully reduced by siRNA $(\mathrm{P}<0.01$; Fig. 6$)$.

MTT assay revealed that EGF promoted cell growth; however, the cells with a reduced expression of CO-029 proliferated at a lower rate compared with the cells transfected with the scramble control siRNA (Scr) in response to EGF treatment (Fig. 7).

We also examined whether CO-029 is involved in the effect of EGF on cell invasion. CO-029 siRNA was transfected into the cells for $24 \mathrm{~h}$, and the cells were subsequently

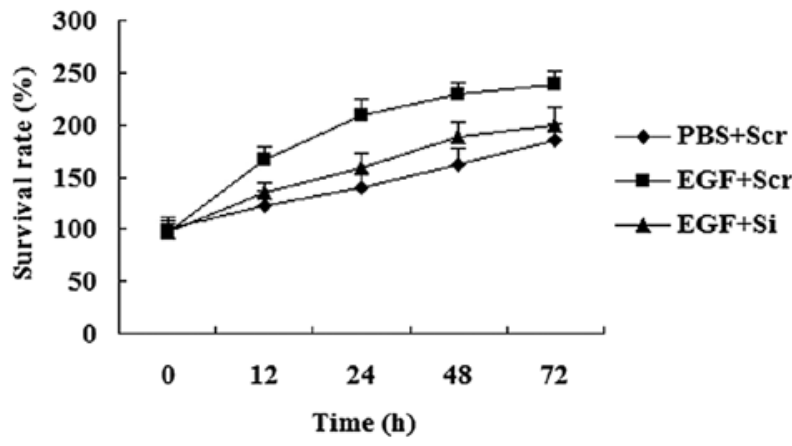

Figure 7. Suppression of CO-029 expression attenuates the effect of epidermal growth factor (EGF) on cell proliferation. $\mathrm{Si}, \mathrm{CO}-029$ siRNA; Scr, scramble control siRNA.

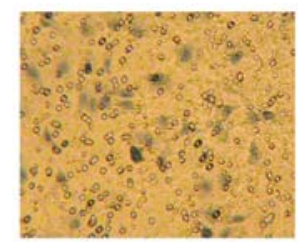

PBS+Scr

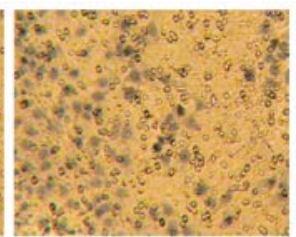

$\mathrm{EGF}+\mathrm{Scr}$

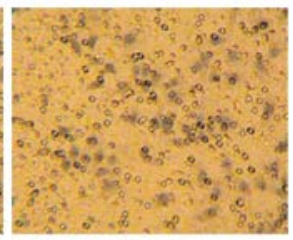

$\mathrm{EGF}+\mathrm{Si}$

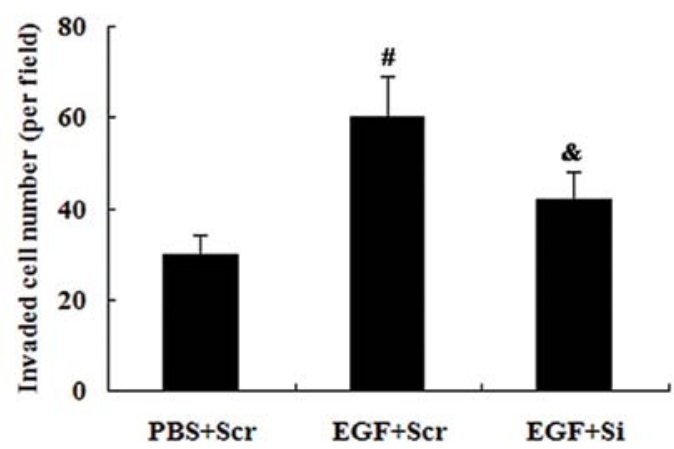

Figure 8. Suppression of CO-029 expression attenuates the effect of epidermal growth factor (EGF on cell invasion. Magnification, $\mathrm{x} 400$. Si, CO-029 siRNA; Scr, scramble control siRNA. ${ }^{*} \mathrm{P}<0.01$ vs. $\mathrm{PBS}+\mathrm{Scr}$ group; ${ }^{\&} \mathrm{P}<0.05$ vs. EGF + Scr group.

treated with $100 \mathrm{ng} / \mathrm{ml}$ EGF for a further $24 \mathrm{~h}$. The results from the Transwell-Matrigel invasion assay revealed that the EGF-induced cell invasion was attenuated by the knockdown of $\mathrm{CO}-029$ expression ( $\mathrm{P}<0.05$; Fig. 8).

\section{Discussion}

In the study by Matsumura et al, it was reported that 53 genes were upregulated in advanced gastric cancer by microarray analysis, and CO-029 was among these upregulated genes (24). In the present study, RT-qPCR and western blot analysis were used to assess the expression of CO-029 in human normal gastric tissues, gastric cancer and tumor-adjacent tissues. We observed that the expression of CO-029 was increased both at the mRNA level and protein level in gastric cancer tissues in comparison to normal gastric tissues and tumor-adjacent tissues. These data indicate that $\mathrm{CO}-029$ acts as an oncogene in human gastric cancer.

Next, we performed in vitro experiments to further investigate the role of $\mathrm{CO}-029$ in gastric cancer cell proliferation and 
invasion. Previous studies have suggested that EGF acts as a mitogen in gastrointestinal tissue and stimulates epithelial cell proliferation, differentiation and growth $(25,26)$. It has been demonstrated that EGF increases mucus synthesis in vitro, and during the epithelial repair process, EGF aids the proliferating cells to migrate into the superficial epithelium (27). The EGFR pathway appears to play a crucial role in the progression of gastric cancer. The expression of EGF and its receptor has been found to correlate with the prognosis of patients with gastric cancer $(28,29)$. In this study, to the best of our knowledge, we investigated for the first time the regulatory effect of EGF on CO-029 expression. The results from western blot analysis revealed that EGF increased the expression of CO-029 in a concentration- and a time-dependent manner. In addition, AGS cells treated with EGF proliferated at a higher rate and showed an enhanced invasion ability.

To determine whether CO-029 is involved in the effects of EGF on gastric cancer cell proliferation and invasion, CO-029 was knocked down by siRNA in the AGS cells. The cells were then treated with EGF. We found that the effects of EGF on gastric cancer cell proliferation and invasion were attenuated by the knockdown of CO-029. These results indicate that CO-029 promotes gastric cancer cell proliferation and invasion mediated by EGF.

Taken together, our study strongly suggests that CO-029 is an oncogene in human gastric cancer. The results from our in vitro experiment demonstrate a specific upregulation of CO-029 in AGS cells treated with EGF, and CO-029 at least partially mediates the effects of EGF on gastric cancer cell proliferation and invasion. It can thus be hypothesized that CO-029 plays an important role in the progression of cancer and may thus emerge as a novel target for therapeutic intervention in gastric cancer.

\section{References}

1. Thun MJ, DeLancey JO, Center MM, Jemal A and Ward EM: The global burden of cancer: priorities for prevention. Carcinogenesis 31: 100-110, 2009.

2. Power DG, Kelsen DP and Shah MA: Advanced gastric cancer-slow but steady progress. Cancer Treat Rev 36: 384-392, 2010.

3. Rohatgi PR, Yao JC, Hess K, et al: Outcome of gastric cancer patients after successful gastrectomy: influence of the type of recurrence and histology on survival. Cancer 107: 2576-2580, 2006.

4. Ott K, Lordick F, Blank S and Büchler M: Gastric cancer: surgery in 2011. Langenbecks Arch Surg 396: 743-758, 2011.

5. Tetzlaff ED, Cheng JD and Ajani JA: Review of docetaxel in the treatment of gastric cancer. Ther Clin Risk Manag 4: 999-1007, 2008.

6. Claas C, Stipp CS and Hemler ME: Evaluation of prototype transmembrane 4 superfamily protein complexes and their relation to lipid rafts. J Biol Chem 276: 7974-7984, 2001.

7. Maecker HT, Todd SC and Levy S: The tetraspanin superfamily: molecular facilitators. FASEB J 11: 428-442, 1997.

8. Todres E, Nardi JB and Robertson HM: The tetraspanin superfamily in insects. Insect Mol Biol 9: 581-590, 2000.

9. Fitter S, Sincock PM, Joliffe CN and Ashman LK: Transmembrane 4 superfamily protein CD151 (PETA-3) associates with beta1 and alpha IIb beta3 integrins in haematopoietic cell lines and modulates cell-cell adhesion. Biochem J 338: 61-70, 1999.

10. Horváth G, Serru V, Clay D, Billard M, Boucheix C and Rubinstein E: CD19 is linked to the integrin-associated tetraspans CD9, CD81, and CD82. J Biol Chem 273: 30537-30543, 1998.
11. Indig FE, Diaz-Gonzales F and Ginsberg MH: Analysis of the tetraspanin CD9-integrin alphaIIbbeta3 (GPIIb-IIIa) complex in platelet membranes and transfected cells. Biochem J 327: 291-298, 1997.

12. Lozahic S, Christiansen D, Manié S, Gerlier D, Billard M, Boucheix C and Rubinstein E: CD46 (membrane cofactor protein) associates with multiple betal integrins and tetraspans. Eur J Immunol 30: 900-907, 2000.

13. Tiwari-Woodruff SK, Buznikov AG, Vu TQ, Micevych PE, Chen K, Kornblum HI and Bronstein JM: OSP/claudin-11 forms a complex with a novel member of the tetraspanin super family and betal integrin and regulates proliferation and migration of oligodendrocytes. J Cell Biol 153: 295-305, 2001.

14. Claas C, Seiter S, Claas A, Savelyeva L, SchwabM and Zöller M: Association between the rat homologue of CO-029, a metastasisassociated tetraspanin molecule and consumption coagulopathy. J Cell Biol 141: 267-280, 1998.

15. Odintsova E, SugiuraT and Berditchevski F: Attenuation of EGF receptor signaling by a metastasis suppressor, the tetraspanin CD82/KAI-1. Curr Biol 10: 1009-1012, 2000.

16. Testa JE, Brooks PC, Lin JM and Quigley JP: Eukaryotic expression cloning with an antimetastatic monoclonal antibody identifies a tetraspanin (PETA-3/CD151) as an effector of human tumor cell migration and metastasis. Cancer Res 59: 3812-3820, 1999.

17. Greco C, Bralet MP, Ailane N, Dubart-Kupperschmitt A, Rubinstein E, Le Naour F and Boucheix C: E-cadherin/p120catenin and tetraspanin Co-029 cooperate for cell motility control in human colon carcinoma. Cancer Res 170: 7674-7683, 2010.

18. Zhou Z, Ran YL, Hu H, et al: TM4SF3 promotes esophageal carcinoma metastasis via upregulating ADAM12m expression. Clin Exp Metastasis 25: 537-548, 2008.

19. Herlevsen M, Schmidt DS, Miyazaki K and Zöller M: The association of the tetraspanin D6.1A with the alpha6beta4 integrin supports cell motility and liver metastasis formation. J Cell Sci 116: 4373-4390, 2006.

20. Kanetaka K, Sakamoto M, Yamamoto Y, Yamasaki S, Lanza F, Kanematsu T and Hirohashi S: Overexpression of tetraspanin CO-029 in hepatocellular carcinoma. J Hepatol 35: 637-642, 2001.

21. Sartore-Bianchi A, Bencardino K, Di Nicolantonio F, et al: Integrated molecular dissection of the epidermal growth factor receptor (EFGR) oncogenic pathway to predict response to EGFR-targeted monoclonal antibodies in metastatic colorectal cancer. Target Oncol 5: 19-28, 2010.

22. Lafky JM, Wilken JA, Baron AT and Maihle NJ: Clinical implications of the ErbB/epidermal growth factor (EGF) receptor family and its ligands in ovarian cancer. Biochim Biophys Acta 1785:232-265, 2008

23. Lin JX, Jia YD and Zhang CQ: Effect of epidermal growth factor on follicle-stimulating hormone-induced proliferation of granulosa cells from chicken prehierarchical follicles. J Zhejiang Univ Sci B 12: 875-883, 2011

24. Matsumura N, Zembutsu H, Yamaguchi K, et al: Identification of novel molecular markers for detection of gastric cancer cells in the peripheral blood circulation using genome-wide microarray analysis. Exp Ther Med 2: 705-713, 2011.

25. Ross JS and McKenna BJ: The HER-2/neu oncogene in tumors of the gastrointestinal tract. Cancer Invest 19: 554-568, 2001.

26. Wang L, Wilson EJ, Osburn J and Delvalle J: Epidermal growth factor inhibits carbachol-stimulated canine parietal cell function via protein kinase C. Gastroenterology 110: 469-477, 1996.

27. Ma L, Liu ES, Chow JY, Wang JY and Cho CH: Interactions of EGF and ornithine decarboxylase activity in the regulation of gastric mucus synthesis in cigarette smoke exposed rats. Chin J Physiol 42: 137-143, 1999.

28. Yasui W, Hata J, Yokozaki H, Nakatani H, Ochiai A, Ito H and Tahara E: Interaction between epidermal growth factor and its receptor in progression of human gastric carcinoma. Int J Cancer 41: 211-217, 1988.

29. Jonjić N1, Kovac K, Krasević M, Valković T, Ernjak N, Sasso F and Melato M: Epidermal growth factor-receptor expression correlates with tumour cell proliferation and prognosis in gastric cancer. Anticancer Res 17: 3883-3888, 1997. 